\title{
Correspondence
}

http://dx.doi.org/10.11646/phytotaxa.202.3.7

\section{Corrections to the nomenclature of genus Diatoma (Bacillariophyta: Fragilariaceae)}

\section{SAÚL BLANCO ${ }^{1 *} \&$ DAVID M. WILLIAMS ${ }^{2}$}

${ }^{I}$ Department of Biodiversity and Environmental Management, University of Leon, Leon, Spain. Current address: The Institute of the Environment. La Serna, 58, 24007 Leon, Spain.

${ }^{2}$ Department of Life Sciences, the Natural History Museum, Cromwell Road, London, United Kingdom.

*Corresponding author (sblal@unileon.es)

Within plants, algae and fungi, the rules of nomenclature (ICN, McNeill et al. 2012) require that adjectival specificand infraspecific - epithets agree grammatically (gender concordance) with the generic name. Epithets not conforming to this rule, although validly published, are to be corrected (ICN art. 23.5) without change of the author citation or date. Despite that the original spelling of these epithets is generally to be retained (art. 60.1), improper Latin terminations are always to be changed to accord with, among others, the mentioned art. 23.5 (art. 32.2).

From a philological point of view, the gender of genus Diatoma Bory (1824: 461) is not evident. Generic names derived from Greek which end in $-m a(-\mu \alpha)$ are neuter, unless this is a rendering of $-m \hat{e}(-\mu \eta)$ (Stearn 1973). Christensen (1991) agrees that, despite Greek words formed on -о $\mu \alpha$ are neuter, Diatoma comes from the Greek $\Delta l \alpha \tau o \mu \eta$ (which means "incision" or "cut in half"), and these Greek words ending in -o $\mu \eta$ — which gives -oma in Latin—are feminine. Nicolson (1994) states explicitly that Diatoma is feminine (see also Zabinkova 1965).

600

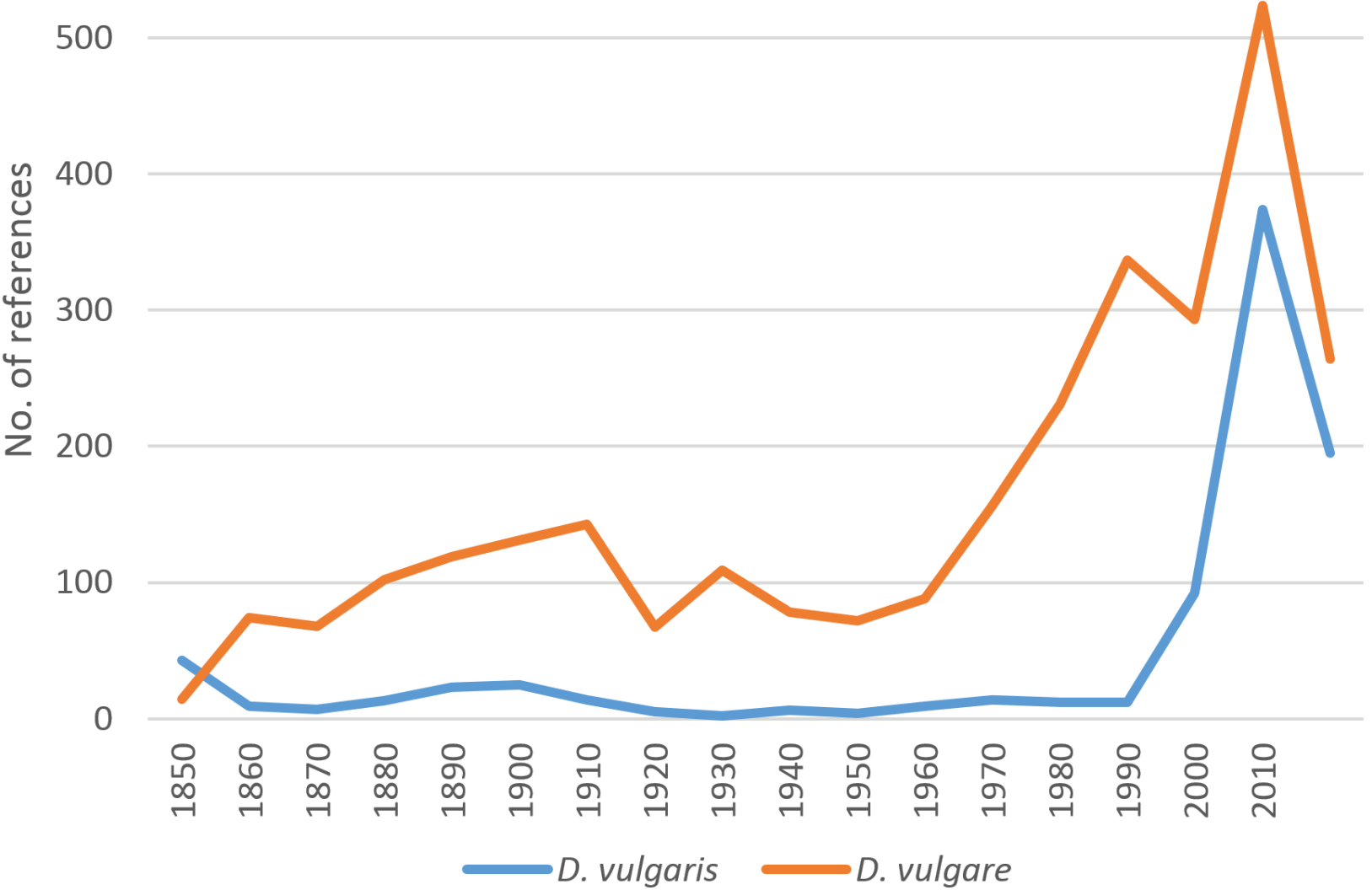

FIGURE 1. References to 'Diatoma vulgare' and 'Diatoma vulgaris' in the literature since original description (1824), based on Google Scholar and Google Books results. 
Art. 62.1 states that "a generic name retains the gender assigned by nomenclatural tradition, irrespective of classical usage or the author's original usage". For Diatoma, the literature has used predominantly neuter combinations (Hartman 1967). For example, we have observed (fig. 1) that the use of the neuter 'Diatoma vulgare' has been much more frequent than the correct feminine 'Diatoma vulgaris' through the last decades. Although Bory did not designate a generitype, his use of 'Diatoma vulgaris' confirms that he treated Diatoma as feminine, although most subsequent authors did not follow this criterion. As Bory acknowledges (Fourtanier \& Kociolek 1999), he adopted a designation used earlier (Diatoma de Candolle in Lamark 1805: 48) for this genus of araphid diatoms, so that Kanitz (1887) superfluously published the substitute name Neodiatoma Kanitz (1887: 5) to which a few species were transferred. Noteworthy, Diatoma had been used earlier to designate the Rhizophoraceae Diatoma Loureiro (1790: 296), nom. rej. (三Carallia Roxburgh 1811: 8) and, despite de Candolle considering Diatoma as neuter, both Loureiro's Diatoma and Kanitz's Neodiatoma (Guiry \& Guiry 2014) are commonly (and originally) treated as feminine. When Bory's Diatoma was conserved against previous usages (Lanjouw et al. 1961: 208), the proposed conserved type D. vulgaris was intentionally corrected to ' $D$. vulgare', erroneously assuming that the original feminine form was wrong (Christensen 1991). Later (and current) editions of the Nomina generica conservanda corrected it to D. vulgaris. Accoding to art. 14.11, "a name may be conserved in order to preserve a particular spelling or gender", and, despite Diatoma Bory was not preserved for this reason (but to preserve nomenclatural stability), we consider that the publication of $D$. vulgaris as the conserved type of Diatoma Bory, nom. cons. ["Diatoma Bory, Dict. Class. Hist. Nat. 5: 461.15 Mai 1824. Typus: D. vulgaris Bory (typ. cons.)"] confirms also the gender of Diatoma as feminine.

Therefore, we provide hereby a corrected list of commonly referred Diatoma taxa (with adjectival epithets) known to have been used recently with improper terminations. Other combinations may be corrected henceforth likewise:

Diatoma moniliformis (Kützing 1833: 580) D.M. Williams (2012: 260)

Diatoma vulgaris Bory (1824: 461)

Diatoma vugaris var. brevis Grunow (1862: 49)

Diatoma vulgaris var. producta Grunow (1862: 49)

Diatoma vulgaris var. ovalis (Fricke in Schmidt et al. 1874-1944: 268) Lange-Bertalot et al. (1991: 116-117)

Diatoma vulgaris var. linearis Grunow in Van Heurck (1881: 50)

Diatoma tenuis C. Agardh (1812: 15)

\section{References}

Agardh, C.A. (1812) Dispositio Algarum Sveciae. Litteris Berlingianis, Lund. 45 pp. http://dx.doi.org/10.5962/bhl.title.45472

Bory de Saint-Vincent, J.B.G.M. (1824a) diatome. Diatoma. zool. ? bot. crypt. (Arthrodiées). In: Dictionnaire classique d'histoire naturelle, par Messieurs Audouin, Isid. Bourdon, Ad. Brongniart, De Candolle, Daudebard de Férusac, A. Desmoulins, Drapiez, Edwards, Flourens, Geoffroy de Saint-Hilaire, A. De Jussieu, Kunth, G. de Lafosse, Lamouroux, Latreille, Lucas fils, Presle-Duplessis, C. Prévost, A. Richard, Thiébaut de Berneaud, et Bory de Saint-Vincent. Ouvrage dirigé par ce dernier collaborateur, et dans lequel on a ajouté, pour le porter au niveau de la science, un grand nombre de mots qui n'avaient pu faire partie de la plupart des Dictionnaires antérieurs. Tome cinquième CRA-D. Rey et Gravier; Baudoin frères, Paris, pp. 1-461. http://dx.doi.org/10.5962/bhl.title.33901

Christensen, T. (1991) The Diatoms. Biology and Morphology of the Genera. Phycologia 30: 233-235. http://dx.doi:10.2216/i0031-8884-30-2-233.1

Fourtanier, E. \& Kociolek, J.P. (1999) Catalogue of the Diatom Genera. Diatom Research 14: 1-190. http://dx.doi:10.1080/0269249X.1999.9705462

Grunow, A. (1862) Die osterreichischen Diatomaceen nebst Anschluss einiger neuen Arten von anderen Lokalitaten und einer kritischen Uebersicht der bisher bekannten Gattungen und Arten. Erste Folge. Epithemieae, Meridioneae, Diatomeac, Entopyleae, Surirelleae, Amphipleureae. Verhandlungen Der Kaiserlich-Königlichen Zoologisch-Botanischen Gesellschaft in Wien 12: 315-372. http://dx.doi.org/10.5962/bhl.title.64361

Guiry, M.D. \& Guiry, G.M. (2014) AlgaeBase World-wide electronic publication, National University of Ireland, Galway. Available from: http://www.algaebase.org (accessed 2 December 2014).

Hartman, A.A. (1967) Le Genre Diatoma aux Pays-Bas. Phycologia 6: 240-246. 
http://dx.doi:10.2216/i0031-8884-6-4-240.1

Kanitz, Á. (1887) A növényrendszer áttekintése: Systematis vegetabilium janua, in usum auditorum R. Universitatis claudiopolitanae. Második átdolgozott kiadás. Kolozsvárt, pp. 1-6.

Kützing, F.T. (1833) Synopsis Diatomacearum oder Versuch einer systematischen Zusammenstellung der Diatomeen. Linnaea 8 (5): 529-620.

Lamarck, J.B. de M. de (1805) Flore française ou Descriptions succintes de toutes les plantes qui croissent naturellement en France, disposées selon une nouvelle méthode d'analyse, et précédées par un Exposé des principes élémentaires de la botannique. Desray, Paris, 922 pp.

Lange-Bertalot, H., Rumrich, U. \& Hofmann, G. (1991) Zur Revision der Gattung Diatoma Bory (Subgenus Diatoma, Bacillariophyceae). Identifikation ökologisch wichtiger, aber taxonomisch problematischer Arten. Acta Biologica Benrodis 3: 115-130.

Loureiro, J. de (1790) Flora Cochinchinensis, sistens plantas in regno Cochinchina nascentes, quibus accedunt alice observatce in Sinensi Imperio, Africa Orientali, Indiceque locis variis: omnes dispositce secundum systema sexuale linnceanum. Typis, et expensis Academicis, Lisbon, $353 \mathrm{pp}$.

http://dx.doi.org/10.5962/bhl.title.560

McNeill, J., Barrie, F.R., Buck, W.R., Demoulin, V., Greuter, W., Hawkworth, D.L., Herendeen, P.S., Knapp, S., Marhold, K., Prado, J., Prud'homme van Reine, W.F., Smith, G.F., Wiersema, J.H. \& Turland (Eds.) (2012) International Code of Nomenclature for Algae, Fungi, and Plants (Melbourne Code) Regnum Vegetabile 154: 1-208.

Lanjouw, J., Baehni, C., Robyns, W., Ross, R., Rousseau, J., Schopf, J.M., Schulze, G.M., Smith, A.C., Vilmorin, R. de \& Stafleu, F.A. (Eds.) (1961) International Code of Botanical Nomenclature, adopted by the Ninth International Botanical Congress, Montreal, August 1959. Regnum Vegetabile 23: 1-372.

Roxburgh, W. (1811) Plants of the Coast of Coromandel. Bulmer, London, 100 pp. http://dx.doi.org/10.5962/bhl.title.467

Schmidt, A. (1874-1944) Atlas der Diatomaceen-Kunde. R. Reisland, Leipzig, 1976 pp. http://dx.doi.org/10.5962/bhl.title.64396

Stearn, W.T. (1973) Botanical Latin. David \& Charles, Newton Abbot, 566 pp.

Van Heurck, H.F. (1881) Synopsis des diatomées de Belgique: Atlas. Édité par l'auteur, Anvers, 291 pp. http://dx.doi.org/10.5962/bhl.title.1990

Williams, D.M. (2012) Diatoma moniliforme: Commentary, relationships and an appropriate name. Nova Hedwigia Beiheft 141: $255-261$.

Zabinkova, N.N. (1965) Generic names ending in -ma and family names derived from them. Taxon 14: 184.

http://dx.doi.org/10.2307/1217141 